\title{
Teamwork and Healthy Workplaces: Strengthening the Links for Deliberation and Action through Research and Policy
}

\author{
- 0 \\ COMMENTARY \\ Ivy Oandasan, MD, MHSc, CCFP, FCFP \\ Associate Professor and Director \\ The Office of Interprofessional Education \\ University of Toronto \\ $\propto$
}

\begin{abstract}
The two lead articles for this issue by Shamian and El-Jardali and by Clements, Dault and Priest provide an opportunity to consider how two agendas - teamwork in healthcare and the healthy workplace - can be strengthened to gain mutual advancement. Both agendas are in the pan-Canadian Health Human Resource (HHR) strategic plan in Canada and were also identified within the Health Council of Canada's 2005 Annual Report. Strong links have yet to be made related to the teamwork in healthcare agenda and its relationship with the workplace environment. Significant research has been conducted, and advocates are pushing for policy change. It is recommended that those engaged in the research in these two domains dialogue with each other and collectively consider ways in which they could advance the policy directions required to enhance both patient and provider satisfaction in our healthcare system. The teamwork and healthy workplace agendas require thoughtful deliberation between researchers and policy-makers to inform action.
\end{abstract}


This commentary provides an example of how the Ontario government has been able to engage within an evidence-informed process to develop inter-professional care that may ultimately positively impact the teamwork in healthcare agenda and the healthy workplace agenda in the future.

IN THIS ERA of healthcare renewal, collaborative healthcare delivery and teamwork are top of mind for many Canadians and policy decision makers. Research has shown that in certain healthcare settings, healthcare professionals who practise in "teams" in their workplace results in improved provider and patient care outcomes (Canadian Health Services Research Foundation [CHSRF] 2006). Yet, we have not seen teamwork practised in all healthcare settings, nor is it a priority at the system, practice or institutional levels in providing the tools and resources for healthcare professionals to provide collaborative healthcare delivery.

Engaging in teamwork requires that health professionals possess the competencies and skills to practise as collaborators within healthcare teams through education, whether at the pre-licensure, post-licensure or continuing education level. Further, most health professionals need to understand the complexity to engage in teamwork and to know when to collaborate, with whom, how and why. This can be challenging for healthcare professionals who practise in different healthcare settings to define their teams or engage in teamwork.

Romanow noted, "If health care professionals are expected to work in teams ... their education must prepare them to do so or else they will continue to work in status quo health care environments" (Commission on the Future of Health Care in Canada 2002). The challenge is how to translate the education of teamwork to healthcare professionals in the health workplace setting through research and policy.
In the past three years, initiatives have been under way across the country to facilitate teamwork in healthcare environments. Specifically, the implementation of teamwork or inter-professional care is one strategy considered for effective health human resources planning in making the healthcare system more sustainable and cost effective. Progress has been made, but at a gradual pace. It is well known that current professional practices foster a system of separate silos of professional practice and impose major constraints on the development of team-based care. At the government level, legislative and regulatory reforms need to keep up with changes and trends in the practice environment. At the organizational level, the focus has been on the perceived lack of responsiveness and willingness of healthcare groups to change or work together. This, despite the fact that professional groups acknowledge the urgent need for health system renewal and are willing to co-operate. Within institutions, support and funding for inter-professional care is fairly minimal due to the inherent incremental operating and administrative costs involved. At the individual level, there is a need to change prevailing mindsets on how healthcare professions can work together, given the entrenched attitudes and views of health professions' respective roles. The resistance to change must be overcome in order to create a sustainable healthcare workforce.

Building a stable health workforce requires innovative, flexible ways to educate healthcare providers. More importantly, it requires better integration between the 
education system that prepares healthcare providers and the health system that employs and deploys them (Government of Ontario 2005). It is widely recognized that planning for health human resources must be a truly collaborative process because it affects every facet of the healthcare system. The change in the healthcare system must be linked with how health professionals are educated and how they apply what has been learned in the workplace setting.

Health Canada's Interprofessional Education for Collaborative PatientCentred Practice initiative has been one strategy of the pan-Canadian Health Human Resource Strategy as the means to address the challenges with health human resource and workplace issues (i.e., shortages). Funding of over $\$ 20$ million has been awarded to educational leaders across the country to develop ways to enhance health professionals to learn with, from and about each other in providing quality and collaborative patient care delivery. Much has been done to help move this strategy agenda forward through an evidence-informed approach that is influencing public policy. Ultimately, the goal is to improve patient care - but what about the goal for healthcare providers and their workplace environment?

In their article on the healthy workplace agenda, Shamian and El-Jardali describe key drivers that lie at the heart of both the teamwork and healthy workplace agendas. Of significance is that effective teamwork practices will improve the well-being of healthcare providers. Their article summarizes that there is evidence to support that healthy workplaces improve recruitment and retention, workers' health and well-being, quality of care and patient safety, organizational performance and societal outcomes. They note that several studies have shown an inverse relationship between nurse staff- ing and adverse events. Poor workplaces have resulted in a substantial health burden and cost to health service organizations as a result of ill health among their staff, impacting loss time from work, errors and litigation.

Similarly, the literature review of teamwork in healthcare synthesis that was conducted by Oandasan et al. found evidence to support that health professionals working in collaborative teams have increased provider satisfaction in the workplace, resulting in reduced staff shortages and decreasing stress and burnout levels among healthcare professionals (CHSRF 2006). Key factors that led to teamwork lie in leadership, availability of resources and the provision of innovative organizational supports and structures to achieve healthy workplaces. In a recent study conducted by West et al. (2006), the authors emphasize that investing in health human resource systems in hospital settings that develop policies and practices focusing on training, performance management, participation, decentralized decision making, involvement, teams and employment security contributes to high-quality care, including improved patient mortality statistics. The findings suggest that managers and policy-makers should focus on improving the functioning of relevant human resource management systems in healthcare organizations.

The teamwork in healthcare synthesis (CHSRF 2006) and the article by Shamian and El-Jardali support the need to focus on organizational leadership to develop clear organizational philosophies that support teamwork and healthy workplace environments to improve patient care and provider satisfaction. The teamwork synthesis revealed, however, that an investment in resources and organizational structures alone will not foster effective teamwork. Individual willingness and capacity to 
Figure 1. Inter-professional education for collaborative patient-centred practice. Reproduced with permission from D'Amour and Oandasan (2005).

Interprofessional Education for Collaborative Patient-centred Practice: An Evolving Framework

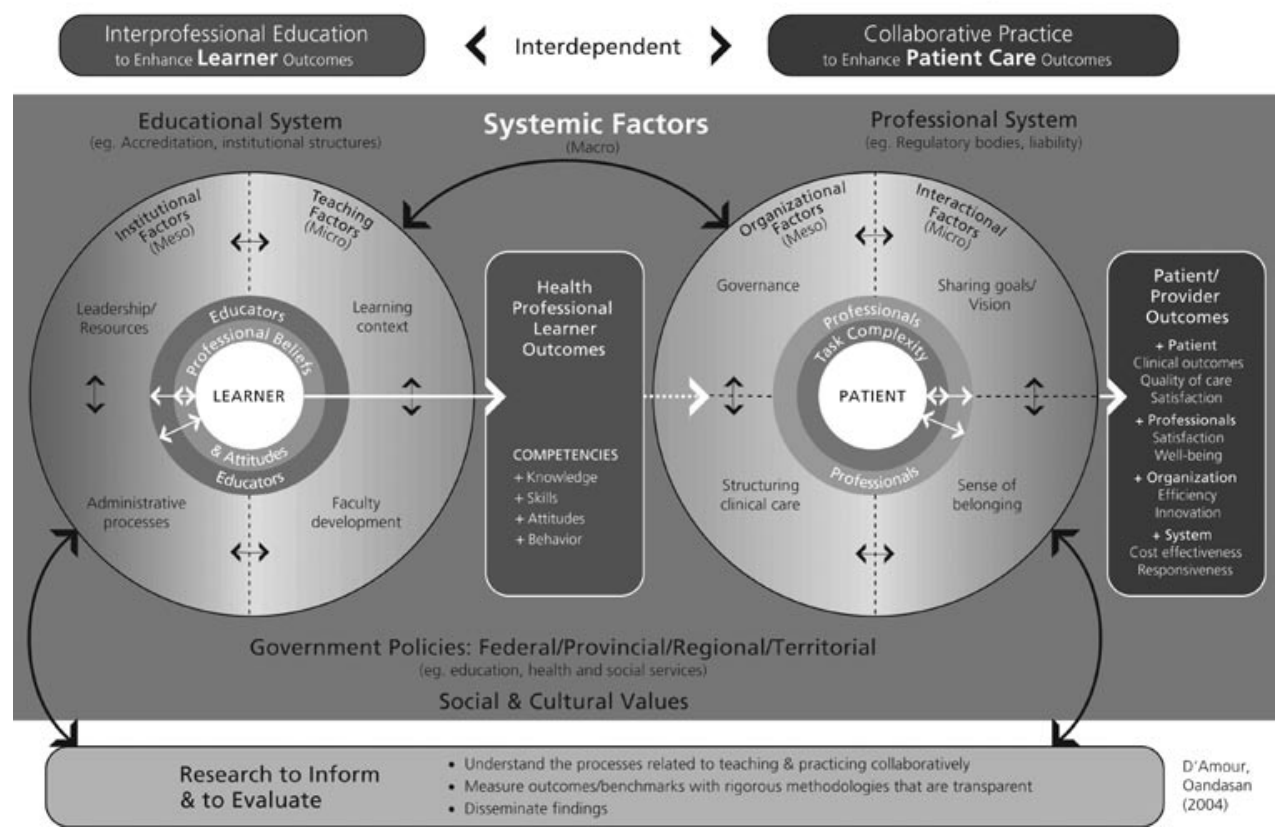

engage in teamwork are needed for success. Attitudinal willingness and capacity or competence to engage in teamwork imply the need for health professionals to be educated through professional development or inter-professional education curricula for those still in their formative years. The teamwork synthesis builds upon the evolving framework that was developed by D'Amour and Oandasan (2005), which concludes that inter-professional education is interdependent with collaborative practice. The framework shown in Figure 1 suggests that one must learn how to be a collaborator in order to practise collaboration within healthcare teams. According to D'Amour and Oandasan, it is therefore the responsibility of educators to teach the competencies of collaboration to learners so that they can enter the workforce when they graduate applying principles and competencies related to collaboration in their workplaces.

However, many healthcare organizations are not structured in a way that supports teamwork in the workplace. This has led to unhealthy work environments. For policymakers, the ultimate goal of teamwork is the improvement of patient care outcomes, the enhancement of provider satisfaction and the advancement of organizational and system efficiencies. Further research is needed to support the argument that healthy workplaces through teamwork will enhance recruitment and retention and patient care. Yet, an opportunity exists to study this area if we believe that teamwork and healthy workplaces are important for health system reform. We need a better understanding of if, how and why teamwork and healthy workplaces can positively impact the delivery of care. This evidence can inform the development of public policies. But, is 
Table 1. Priorities arising from the Ontario summit to advance inter-professional care Education and Research

1. Incorporate required inter-professional care curricula by establishing appropriate learning strategies and timelines.

2. Agree upon shared competencies and education models, based on evidence (i.e., demonstration projects) and incorporate them into curricula, faculty development, clinical education and accreditation.

3. Use innovative technologies to educate and engage health professionals and consumers in inter-professional care.

4. Evaluate educational models developed to ensure sustainability - use demonstration models and share learnings.

5. Implement mechanisms to educate and engage health professionals and consumers alike about inter-professional care.

Regulation and Liability

1. Define inter-professional care practices in healthcare settings that currently do not require regulatory changes.

2. Develop definitions and standards for scope of practice and core competencies for all healthcare professions that can be applied to all healthcare settings.

3. Implement mandatory adequate liability protection for collaborative care practices and settings.

4. Encourage regulators to develop collaborative regulations, first establishing a baseline from which to proceed.

5. Address risk management issues that will facilitate inter-professional care.

Organizational Structure

1. Address structural issues that reinforce power hierarchies across healthcare professions.

2. Create champions to facilitate inter-professional communication and leadership development in teamwork.

3. Create incentives for all health professionals to practise collaboratively (within and across sectors).

4. Clarify roles of all players in the healthcare system, including patients.

5. Increase profile, recognition, systemic support and coordination of inter-professional care at all levels to the degree necessary to affect change in the long term.

Cultural Shift

1. Organizational structures, systems and processes (i.e., rewards, incentives, performance appraisals, standards and accreditation) must change to support inter-professional care.

2. Evaluation and continuous improvement are necessary to ensure successful implementation of inter-professional care.

3. Continuous and sustainable funding must be made available for inter-professional care.

4. Information should be broadly shared, and role models (leaders and mentors) should be seen as champions of change toward inter-professional care.

5. Evaluation and creation of standardized indicators will be needed to ascertain when the culture has shifted and to track quality improvement.

there enough evidence to move it forward? Shamian and El-Jardali and Oandasan et al. (CHSRF 2006) believe that there is; however, there is a need to move forward with caution and rigour through evaluation to inform policy decisions.

In June 2006, by invitation, 110 decision makers, healthcare providers, community leaders, researchers and educators gathered in Toronto for the Summit on Advancing Interprofessional Education and Practice. Sponsored by the Ontario government, it sought the input and guidance of summit participants in developing and implementing practical, timely ways to remove the barriers that prevent effective use of health human resources and inter-professional care based upon the evidence for inter-profes- 
sional care collected to date. There was a high level of interest and commitment generated among participants to advance inter-professional care in Ontario. Summit participants identified priorities on action steps for policy development to achieve inter-professional care. These are outlined in Table 1.

As a result of the recommendations that were forwarded by stakeholders at the summit, the Ontario government has provided resources and support to carry out the development of a policy blueprint for advancing inter-professional care in Ontario by spring 2007. The Interprofessional Care Project, which is being carried out by a steering committee and three working groups, intends to build upon the research evidence to inform the policy direction. The steps following the June summit in Ontario provide a living example of how evidenceinformed policy development can be enacted.

Drawing upon the literature review and work that has been done to date, Lomas et al. (2005) have suggested that research evidence can assist in informing policy decisions through a process of deliberation with key stakeholders. Lomas et al. describe three types of evidence: context-specific evidence, context-free evidence (e.g., from randomized control trials) and colloquial evidence. Each has its merits, but collectively the opportunity to make evidencebased informed decisions can be made real by facilitating dialogue among stakeholders - colloquial evidence meets context-specific and context-free evidence from the literature and brings rigour to the development of policy decisions.

Those engaged in enhancing teamwork in practice could learn about the policy interventions that have been implemented over the years on the healthy workplace agenda according to Shamian and El-
Jardali. Policy interventions include public reporting measures, hospital accountability agreements, healthy workplace objectives embedded within strategic plans, the development of accreditation and workplace indicators, more educational seats, new staffing protocols and workload targets. Yet, the authors note that more work is needed to ensure that these policy initiatives bring effective changes to the workplace for better working conditions for healthcare workers. Similar to the recommendations by the teamwork synthesis (CHSRF 2006), the need for collaboration among researchers, policy-makers, decision makers, stakeholders and practitioners is required to attain healthy workplaces.

\section{References}

Canadian Health Services Research Foundation. 2006. Teamwork in Healthcare: Promoting Effective Teamwork in Healthcare in Canada. Ottawa: Author.

Commission on the Future of Health Care in Canada. 2002. Building on Values: The Future of Health Care in Canada. Ottawa: Author. <http://www. hc-sc.gc.ca/english/care/romanow/hcc0086.html>. Accessed December 1, 2006.

D'Amour, D. and I. Oandasan. 2005.

"Interprofessionality as the Field of Interprofessional Practice and Interprofessional Education: An Emerging Concept." Journal of Interprofessional Care 19(Suppl. 1): 8-20.

Government of Ontario. 2005. Laying the Foundation for Change. A Progress Report on Ontario's Health

Human Resources Initiatives. Toronto: Author. $<$ http://www.health.gov.on.ca/english/public/pub/ ministry_reports/hhr_05/hhr_05.html>. Accessed December 1, 2006.

Lomas, J., T. Culyer, C. McCutcheon, L. McAuley and S. Law. 2005. Conceptualizing and Combining Evidence for Health System Guidance: Final Report. Ottawa: Canadian Health Service Research Foundation.

West, M.A., J.P. Guthrie, J.F. Dawson, C.S. Borrill and M. Carter. 2006. "Reducing Patient Mortality in Hospitals: The Role of Human Resource Management." Journal of Organizational Behaviour 27: 983-1002. 